\title{
Study on Dynamic Guidance Obstacle Avoidance for UAV
}

\author{
Xiong Zhiyong ${ }^{1}$, Yang Xiuxia ${ }^{2}$, Zhang $\mathrm{Yi}^{2}$, Hua Wei2 and Zhou Weiei ${ }^{2}$ \\ ${ }^{1}$ Science and Technology on Avionics Integration Laboratory, Shanghai, \\ 200233, China \\ ${ }^{2}$ Department of Control Engineering, Naval Aeronautical and Astronautical \\ University, Yantai, China \\ yangxiuxia@126.com
}

\begin{abstract}
With the flight environment becoming more and more complex, how to avoid the moving threat for the UAV under the dynamic environment and complete the mission become a key problem. With the idea of guidance, using the method of dimension reduction, the dynamic guidance obstacle avoidance method in three dimensional spaces is given. Considering the collision avoidance completion time and the UAV's maneuverability, the parameters range of obstacle avoidance navigation law are deduced. Simulation results show the validity of the algorithm.
\end{abstract}

Keywords: Three dimensional space; Proportional navigation law; UAV; Obstacle avoidance time; Maneuverability.

\section{Introduction}

Currently, UAVs have been widely used in military and civilian fields. How to make the UAV safely guide to the target and successfully avoid the dynamic obstacles has become a hot topic on which many scholars study. O.Khatib [1], Erdmann, LozanoPerez [2] and so on are the earliest scholars who have carried out the research on the problem. Later, scholars extend the path planning and obstacle avoidance methods which have been successfully applied in the static environment to the dynamic environment, for example deterministic and probabilistic roadmap methods are used in [3]; and in [4], by adding the velocity of the robot and the obstacle into the structure of the potential field function, the authors obtain the potential field function that is applicable to the dynamic environment; in [5], a dynamic window approach is used. All these methods perform well in the static environment but not also in the dynamic environment, especially in the case that the UAV has high speed. This is due to the major differences between the collision detection and the control of the velocity.

To solve the problem of collision detection in the dynamic environment, the references [6] and [7] propose a collision detection method that is based on the kinematics equations. In [6] the authors define the concept of collision cone and according to the angle of the relative velocity to judge that there is collision or not. In [7] based on the equation of the angle rate of the sight line, the authors compare the direct collision with the indirect collision.

Taking into account the velocity change of the obstacle in the dynamic environments, scholars have proposed the concept of velocity obstacle. For example, in [8] considering the velocity of the moving obstacles, the authors built the collision cone, and on this basis, by transferring the velocity of the robot to the outside of the cone, the robot can avoid the moving obstacle successfully. But the velocity of the obstacle is required be linear. In [9], the nonlinear velocity obstacle approach is an extended concept of the velocity obstacle to cope with the condition that the obstacles move along the arbitrary trajectories. The nonlinear velocity obstacle can be generated as a time 
integration of the collision velocity. Taking into account the problem of collision avoidance with multi-UAVs, [10] proposes the concept of reciprocal velocity obstacle to solve the problem.

But all of the methods above do not consider the completion time of the collision avoidance process. In fact, it is necessary that the completion time of the collision avoidance process should be less than the time that the UAV reaches the obstacle to ensure the success of the collision avoidance. Therefore, based on the consideration of the completion time of the collision avoidance, this paper presents a collision avoidance algorithm based on the sliding-mode variable structure finite-time convergence guidance law. First, we set up the kinematics equations of the UAV with the obstacles in the polar coordinate and build up the collision cone, then under the guidance law, we design the finite-time convergence collision avoidance algorithm. In the following sections, we take the analysis of system stability and the convergence time, then we know that under the suitable parameters, the convergence time is less than the reaching time to ensure the success of the collision avoidance. Finally, we take the simulation of the algorithm, and the simulation results show the effectiveness of the algorithm.

\section{Collision Avoidance in Three Dimensional Space}

Under actual situations, the movement of UAV is not in two dimensional space only, and the navigation environment of which is three dimensional space. So the study of collision avoidance in three dimensional space has actual significance. In this paper, we suggest to solve the problem of collision avoidance of the UAV in three dimensional space by generalizing the method mentioned above to the three dimensional space.

\subsection{Plane Decomposition}

The Geometric relationship of the UAV and the obstacle is shown as Figure 1 when the UAV is in three dimensional space.

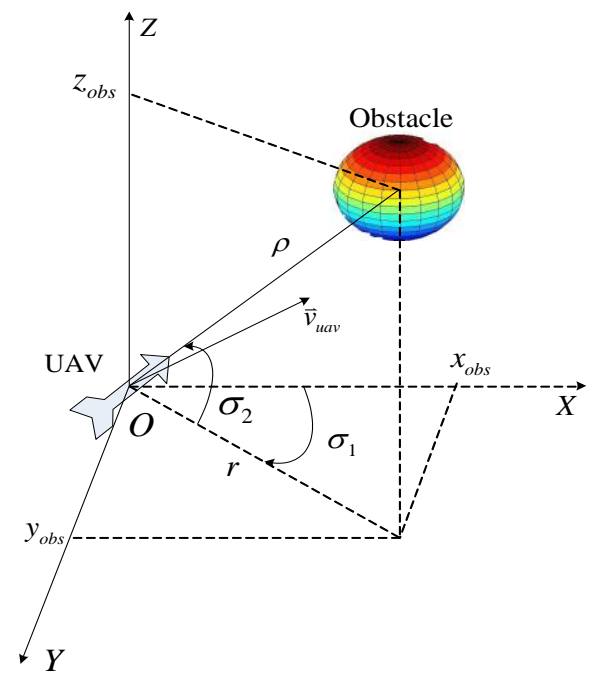

Figure 1. Geometric Relationship of the UAV and the Obstacle in Three Dimensional Space

Where the $\mathrm{X}, \mathrm{Y}$ axis is horizontal axis and the $\mathrm{Z}$ axis is vertical axis. We set that the UAV is at the original point $O$, the coordinate of obstacle is 
$\left(x_{o b s}, y_{o b s}, z_{o b s}\right), \rho$ is the Euclidean distance of the UAV to the center of the obstacle, $r$ is the component of $\rho$ in horizon plane $X O Y$. Now let us decompose three-dimensional space into horizon plane $X O Y$ and vertical plane $r O Z$, as is shown in the Figure 2.Therefore, we know that $\sigma_{1}$ is the horizon line of sight angle to the centre of obstacle-circle, $\sigma_{2}$ is the vertical line of sight angle.

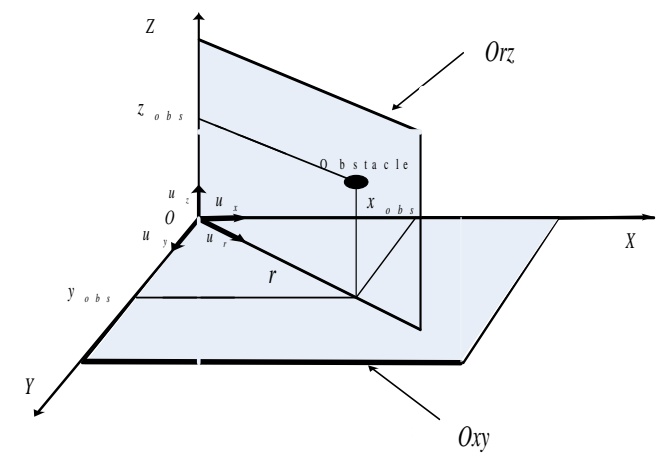

Figure 2. Schematic of the Horizontal Plane and Vertical Plane

Next we can get that the projection of the UAV's speed $\vec{v}_{\text {uav }}$ in horizon plane $X O Y$ is $\vec{v}_{\text {uavxy }}$. So we decompose the speed $\vec{v}_{\text {uav }}$ based on the component $\vec{v}_{\text {uavxy }}$ and $\mathrm{X}, \mathrm{Y}, \mathrm{Z}$ axis, and set up velocity vector level $X O Y$ and velocity vector vertical $\vec{v}_{\text {uavxy }} O Z$.

Therefore we can get that the heading angle of UAV is $\psi_{\text {uav }}$ and the flight path angle is $\psi_{\text {uav }}$, as is shown in the Figure 3, where $v_{\text {uavxy }}=v_{\text {uav }} \cos j_{\text {uav }}$.

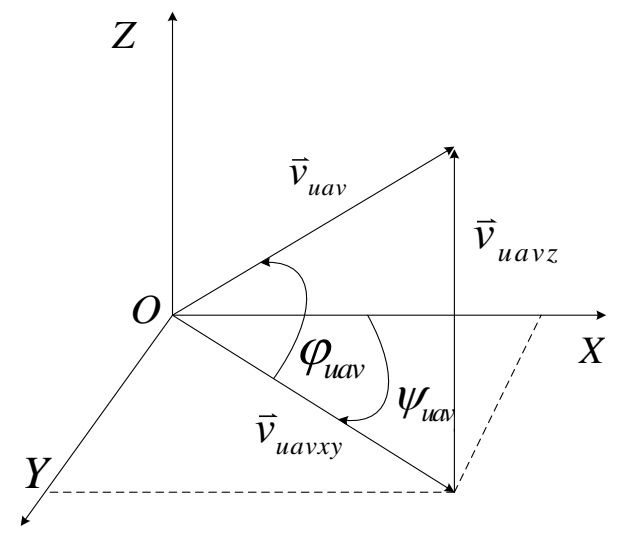

Figure 3. Schematic of the Heading Angle and the Flight Path Angle

\subsection{Collision Estimation}

When knowing that there is a obstacle ahead of the UAV in three dimensional space, we need judge the possibility of collision between the UAV with an obstacle.

If so, the UAV need take measures to avoid the obstacles, the judging method can refer the paper [8]. 
Suppose the UAV and the obstacle are globe, we simplify the UAV as a mass point by expanding the radius of the obstacle globe to $R_{P}=r_{o b s}+r_{u a v}$. Next we get the obstacle circle $O_{x y}$ on the horizontal plane and the one on the vertical plane. Finally we check out whether the heading angle $\psi_{\text {uav }}$ of the UAV is in the obstacle cone of $O_{x y}$ and the flight path angle $\varphi_{u a v}$ is in the obstacle cone of $O_{r z}$.

If both meet, there will be a collision between the UAV and an obstacle after a period of time, the schematic of collision between the UAV and the obstacle in three-dimensional space is shown as Figure 4.
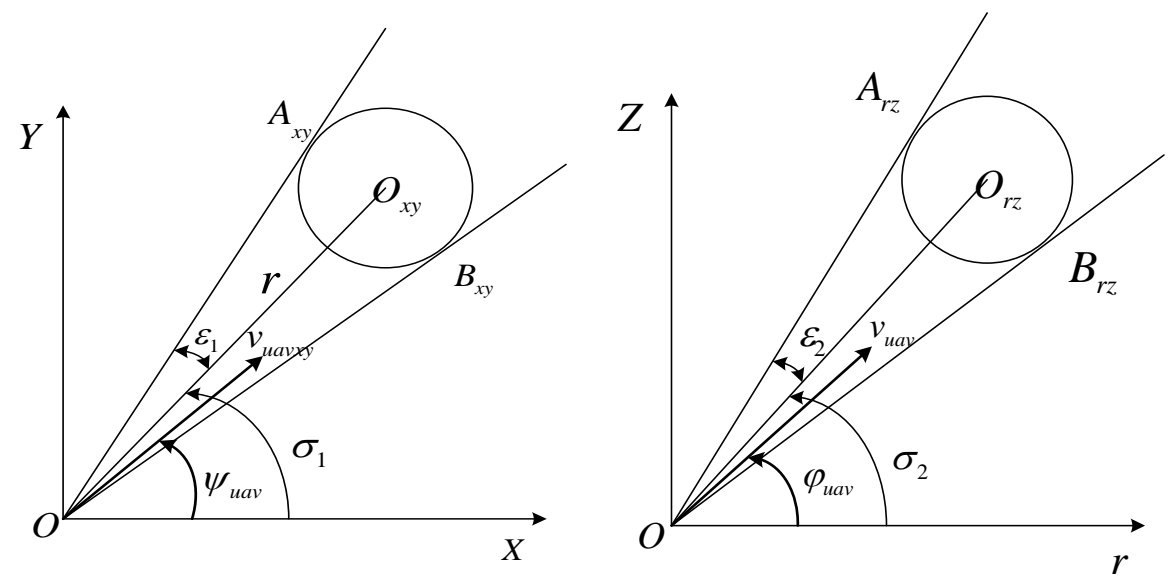

Figure 4. Schematic of Collision between UAV and the Obstacle in Threedimensional Space

Therefore, if there is a collision between the UAV and an obstacle, the formulation (1) and (2) need both meet.

$$
\begin{aligned}
& \sigma_{1}-\varepsilon_{1}<\psi_{\text {uav }}<\sigma_{1}+\varepsilon_{1} \\
& \sigma_{2}-\varepsilon_{2}<\varphi_{\text {uav }}<\sigma_{2}+\varepsilon_{2}
\end{aligned}
$$

\subsection{Collision Avoidance Strategy Based on Proportional Guidance}

From the relationship between proposition and anti-proposition, we know the UAV can avoid obstacle successfully as long as either one if (1) or (2) doesn't meet. Therefore, compare the size of the angle $\psi_{u a v}$ and $\psi_{u a v}$ changes, and $O B_{x y}$ or $O B_{r z}$ is selected as collision avoidance vector based on Figure 4.

\section{(1) OXY plane obstacle avoidance}

If change the heading angle $\psi_{\text {uav }}$ only, we can complete collision avoidance based on the strategy proposed in chapter 2.3 directly. While relative speed $v_{r e l}$ is in the obstacle cone $A U B$, then we need adjust the direction of relative speed $v_{\text {rel }}$ to the direction of collision avoidance vector $U B$ to complete collision avoidance. Now we conduct collision avoidance guidance based on the proportional guidance strategy. The geometric relationship of the UAV and obstacle is shown as Figure 5.From which, we can get the acceleration instruction of relative speed is

$$
a=v_{\text {rel }} \dot{\psi}_{\text {rel }}
$$

And we can get the following result based on the proportional guidance law. 


$$
\dot{\psi}_{r e l}=N \dot{\theta}
$$

Therefore,

$$
a=N v_{r e l} \dot{\theta}_{r e l}
$$

In which, $a_{\text {is }}$ the input acceleration of relative speed, $v_{r e l}$ is the size of relative speed, $\dot{\psi}_{r e l}$ is the angular speed of relative speed, $\dot{\theta}_{\text {is }}$ the angular speed of collision avoidance vector, $N$ proportional navigation coefficient. So if we want to get the acceleration instruction, the angular speed of collision avoidance vector $\dot{\theta}$ must be got, as shown in Figure 5 .

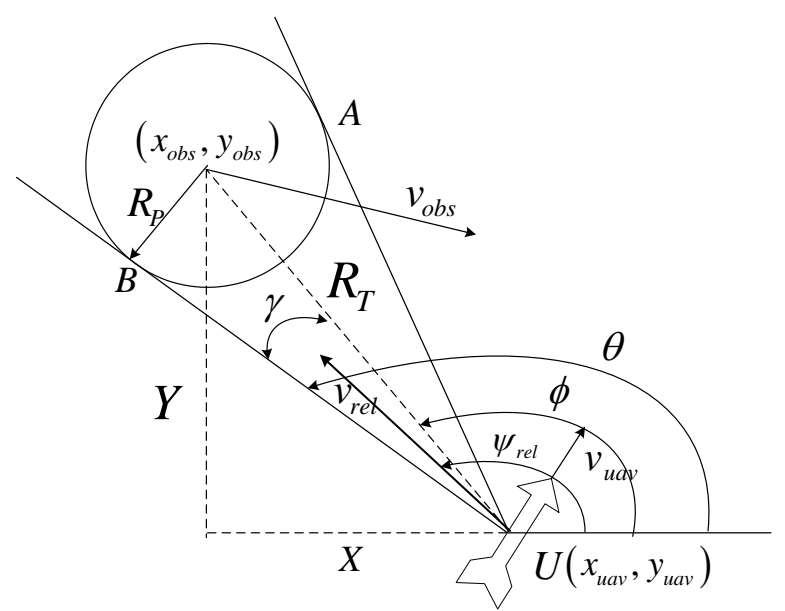

Figure 5. Geometric Relationship of the Collision Avoidance Vector Angle

From Figure 5, we can get,

$$
\begin{gathered}
\sin \phi=\frac{Y}{R_{T}} \cos \phi=\frac{X}{R_{T}} \\
\sin \gamma=\frac{R_{P}}{R_{T}} \\
\dot{Y}=-v_{r e l} \sin \psi_{r e l} \\
\dot{\theta}=\dot{\phi}+\dot{\gamma}
\end{gathered}
$$

In which, $X 、 Y$ is the difference of coordinate value between obstacle and $\mathrm{UAV}$, that is,

$$
X=x_{\text {obs }}-x_{\text {uav }}, Y=y_{\text {obs }}-y_{\text {uav }}
$$

$R_{T}$ is the distance between obstacle and UAV, $\gamma_{\text {is }}$ the included angle of collision avoidance vector and sight line, ${ }^{R_{p}}$ is the radius of expanded obstacle circle.

From the time ${ }^{t}$ derivative of formula(6)、(7)combined with formula(8)、(9), we can get

$$
\dot{\theta}=-\left(\frac{v_{r e l} \sin \psi_{r e l}}{R_{T} \cos \phi}+\frac{\dot{R}_{T}}{R_{T}}(\tan \phi+\tan \gamma)\right)
$$

From paper [8],we can know that to guarantee the relative speed is converged to collision avoidance vector, the proportional navigation coefficient must 
meet $N>1$, which confirms the lower bound of proportional navigation coefficient $N$.

\section{(2) ORY plane obstacle avoidance}

Similarly, if change the flight path angle $\varphi_{\text {uav }}$ only, the geometric relationship of the collision avoidance vector $O B_{r z}$ angle $\theta$ on the vertical plane is shown in Figure 2-15 based on Figure 6.

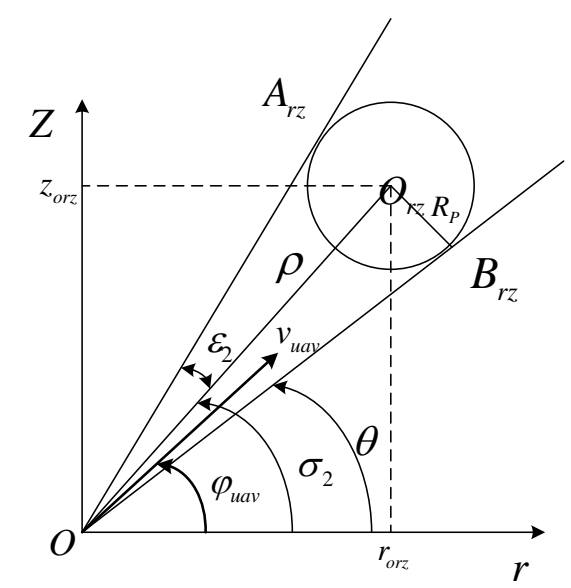

Figure 6. Geometric Relationship of the Collision Avoidance Vector Angle on the Vertical Plane

From Figure 6, we get the following result

$$
\begin{gathered}
\sin \sigma_{2}=\frac{z_{\text {orz }}}{\rho} \cos \sigma_{2}=\frac{r_{\text {orz }}}{\rho} \\
\sin \varepsilon_{2}=\frac{R_{P}}{\rho} \\
\dot{z}_{\text {orz }}=-v_{\text {uav }} \sin \varphi_{\text {uav }} \\
\dot{\theta}=\dot{\sigma}_{2}-\dot{\varepsilon}_{2}
\end{gathered}
$$

Where, $\sigma_{2}$ is the vertical line of sight angle to the centre of obstacle-circle, $\rho$ is the Euclidean distance of the UAV to the center of the obstacle, $R_{p}$ is the radius of the obstacle globe, $\varphi_{\text {uav }}$ is the flight path angle, $\varepsilon_{2}$ is half-cone angle, $z_{o r z}$ is the difference between the coordinate of the obstacle $z_{o b s}$ with the coordinate of the UAV $z_{\text {uav }}$, that

$$
z_{\text {orz }}=z_{\text {obs }}-z_{\text {uav }}
$$

Suppose that the UAV is at the original point, then $z_{\text {orz }}=z_{\text {obs }} \cdot r_{\text {orz }}$ is the

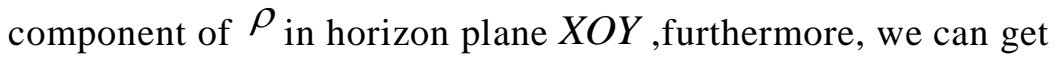

$$
r_{o r z}=\sqrt{\left(y_{o b s}-y_{u a v}\right)^{2}+\left(x_{o b s}-x_{u a v}\right)^{2}}
$$

Substitute equation (13) into equation (14) based on the time $t$ derivation of the formulation(11)、(12), we obtain 


$$
\dot{\theta}=-\left(\frac{v_{u a v} \sin \varphi_{\text {uav }}}{\rho \cos \sigma_{2}}+\frac{\dot{\rho}}{\rho}\left(\tan \sigma_{2}-\tan \gamma\right)\right)
$$

Next we work $\dot{\rho}$ out,

From formulation $\rho=\sqrt{r_{\text {orz }}^{2}+\left(z_{\text {obs }}-z_{\text {uav }}\right)^{2}}$ and the derivation of it, we can get

$$
\dot{\rho}=\frac{\dot{r}_{o r z} r_{o r z}+\left(\dot{z}_{o b s}-\dot{z}_{u z v}\right)}{\rho}
$$

From figure 4, we know

$$
\dot{r}_{\text {orz }}=-v_{\text {uavxy }} \cos \left(\sigma_{1}-\psi_{\text {uav }}\right)
$$

And we know $v_{\text {uavxy }}=v_{\text {uav }} \cos \varphi_{\text {uav }}$, substitute it into (19), we obtain

$$
\dot{r}_{\text {orz }}=-v_{\text {uav }} \cos \varphi_{\text {uav }} \cos \left(\sigma_{1}-\psi_{\text {uav }}\right)
$$

Set obstacle as a stationary point, then

$$
\dot{z}_{\text {obs }}=0, \quad \dot{z}_{\text {uav }}=v_{\text {uav }} \sin \varphi_{\text {uav }}
$$

Substitute equation(20)、(21) into equation(18),we obtain

$$
\dot{\rho}=-v_{\text {uav }}\left[\cos \varphi_{\text {uav }} \cos \left(\sigma_{1}-\psi_{\text {uav }}\right) \cos \sigma_{2}+\sin \varphi_{\text {uav }} \sin \sigma_{2}\right]
$$

Then we can work out the collision avoidance vector $O B_{r z}$ on the vertical plane and the angular velocity $\dot{\theta}$, Finally, based on the proportional navigation guidance law, we design $\dot{\varphi}_{u a v}=N \dot{\theta}$ and guide the flight path angle $\varphi_{\text {uav }}$ of the UAV to the direction of the collision avoidance vector $O B_{r z}$ on the vertical plane to complete collision avoidance.

The selection principle of proportional navigation coefficient can refer to paper [9],taking the collision avoidance of UAV in the plane OXY as an example, when we know the distance $R(0)$ of initial collision avoidance vector, the initial collision avoidance vector angle $\theta(0)$, the relative speed angle $\psi_{\text {rel }}(0)$, the size of relative speed, the proportional navigation coefficient $N$ and minimal value $\varepsilon$, we can get the collision avoidance time $\tilde{t}_{t_{f}}$ based on (23).

$$
\begin{aligned}
\tilde{t}_{t_{f}}= & \frac{R(0)}{v_{r e l}} \int_{\left(\frac{\varepsilon}{\sin \eta(0)}\right.}^{1} \frac{1}{N-1}\left(1+\frac{1}{2} \sin ^{2} \eta(0) \chi^{2(N-1)}\right) d \chi \\
= & \frac{R(0)}{v_{r e l}}\left(1+\frac{1}{2(2 N-1)} \sin ^{2} \eta(0)-\left(\frac{\varepsilon}{\sin \eta(0)}\right)^{\frac{1}{N-1}}\right. \\
& \left.-\frac{1}{2(2 N-1)} \sin ^{2} \eta(0)\left(\frac{\varepsilon}{\sin \eta(0)}\right)^{2+\frac{1}{N-1}}\right)
\end{aligned}
$$

Likewise, we can get the bound of the proportional navigation coefficient $N$ under the initial condition when the collision avoidance time of UAV is given based on (23).

From (23) we know that, the larger the proportional navigation coefficient $N$, the shorter the collision avoidance completing time. In the meanwhile, the larger the normal acceleration of relative speed. But in actual situation, the normal acceleration of UAV can not infinite.

When UAV carries on a uniform fight in the relative speed $v_{\text {rel }}$, the angular speed of collision avoidance vector will approach zero gradually, if the 
proportional navigation coefficient $N>2$. Therefore, the normal acceleration of relative speed $a=v_{\text {rel }} \dot{\psi}_{\text {rel }}=N v_{\text {rel }} \dot{\theta}$ will also approach zero gradually.

From obstacle avoidance algorithm, we can get the relation of acceleration, angular speed and $a(t)$ as following,

$$
\begin{gathered}
\left|\dot{v}_{\text {uav }}(t)\right|=\left|a(t) \sin \left(\psi_{\text {rel }}(t)-\psi_{\text {uav }}(t)\right)\right| \\
\left|\dot{\psi}_{\text {uav }}(t)\right|=\left|a(t) \cos \left(\psi_{\text {rel }}(t)-\psi_{\text {uav }}(t)\right) / v_{\text {uav }}(t)\right|
\end{gathered}
$$

Then we can get the normal acceleration of UAV,

$$
a_{\text {uav }}=\left|v_{\text {uav }}(t) \dot{\psi}_{\text {uav }}(t)\right|=\left|a(t) \cos \left(\psi_{\text {rel }}(t)-\psi_{\text {uav }}(t)\right)\right|
$$

The normal acceleration of UAV is largest at the initial time and approaches zero gradually. From overload $n=a_{\text {uav }} / g$, we know the overload is largest at the initial time. When we know the available overload requirements, we can get the bound of proportional navigation coefficient from(5)、(10)、 (26)under the condition of the largest overload.

\section{Simulation Analysis}

Suppose that there is a obstacle as the UAV is in three dimensional space, the initial conditions of the UAV and the obstacle is shown as Table 2-2. the shape is seen as a ball whose radius is $R=1 \mathrm{~km}$ and the obstacle is set as a stationary point.

The permissible load factor of UAV is $n=5$.

Table 1. The Initial Conditions of the UAV and the Obstacle

\begin{tabular}{|c|c|c|c|c|}
\hline & $(x, y, z)$ & $v$ & $\psi$ & $\varphi$ \\
\hline UAV & $(0 \mathrm{~km}, 0 \mathrm{~km}, 0 \mathrm{~km})$ & $244 \mathrm{~m} / \mathrm{s}$ & $137^{\circ}$ & $35^{\circ}$ \\
\hline Obstacle & $(-10 \mathrm{~km}, 10 \mathrm{~km}, 10 \mathrm{~km})$ & $0 \mathrm{~m} / \mathrm{s}$ & $0^{\circ}$ & $0^{\circ}$ \\
\hline
\end{tabular}

From the initial conditions mentioned above, The Euclidean distance of the UAV to the center of the obstacle $\rho=10 \sqrt{3} \mathrm{~km}, r=10 \sqrt{2} \mathrm{~km}$.

Then we can obtain the horizon line of sight angle to the centre of obstaclecircle $\sigma_{1}=135^{\circ}$, the vertical line of sight angle $\sigma_{2}=35.3^{\circ}, \varepsilon_{1}=8.13^{\circ}, \varepsilon_{2}=6.63^{\circ}$.

Substitute the conditions above into formulation (1) and (2), we obtain

$135^{\circ}-8.13^{\circ}<137^{\circ}<135^{\circ}+8.13^{\circ}$

$35.3^{\circ}-6.63^{\circ}<35^{\circ}<35.3^{\circ}+6.63^{\circ}$

So there is a collision between the UAV and obstacle, and the UAV must take some measures to avoid collision.

For the difference between $\psi_{\text {uav } 0}=137^{\circ}$ and $\sigma_{1}+\varepsilon_{1}=135^{\circ}+8.13^{\circ}$ is minor, we plan to avoid collision by altering the heading angle of UAV $\psi_{\text {uav }}$, and define the chosen tangent line on horizon lane as the collision avoidance vector., whose size is $\sigma_{1}+\varepsilon_{1}=135^{\circ}+8.13^{\circ}$.

During the process of collision avoidance, the velocity of the UAV was set as $v_{\text {uav } 0}=244 \mathrm{~m} / \mathrm{s}$ and stay the same, the horizontal velocity $v_{\text {uavxy }}=200 \mathrm{~m} / \mathrm{s}$ remain the 
same for the flight path angle $\varphi_{\text {uav }}=35^{\circ}$ remain the same. and the selection of proportional navigation coefficient $N$ is same as its counterpart in two dimensional space. the larger $\mathrm{N}$, the shorter the completion-time of collision avoidance under the constraint of the maneuver performance.

Given the initial value of the parameters: $v_{\text {uavxy }}=200 \mathrm{~m} / \mathrm{s}$, the horizontal distance between the UAV and the obstacle $r_{0}=14000 m$, the angular deviation $\eta_{0}=6.13^{\circ}$, the maximum normal overload $n=5$, we can obtain that the longest completion-time of collision avoidance $t_{\max } \approx 70 \mathrm{~s}, N_{\min }=2.6, N_{\max }=165$.

(1) The simulation results under the condition of the longest completion-time of collision avoidance and $N_{\min }=2.6$ are as follows.

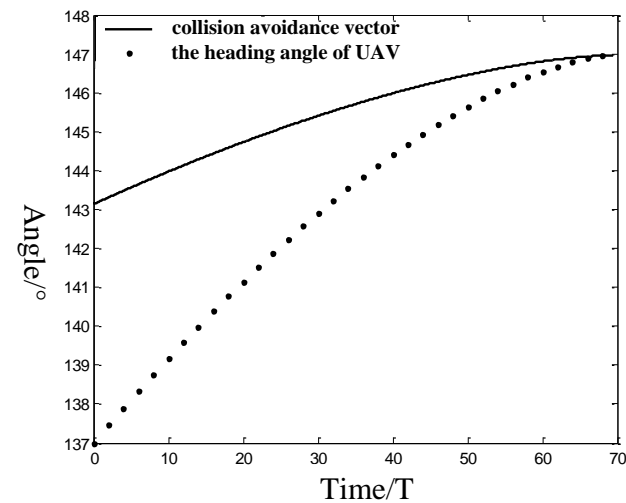

Figure 7. Avoidance Vector Angle and the Heading Angle

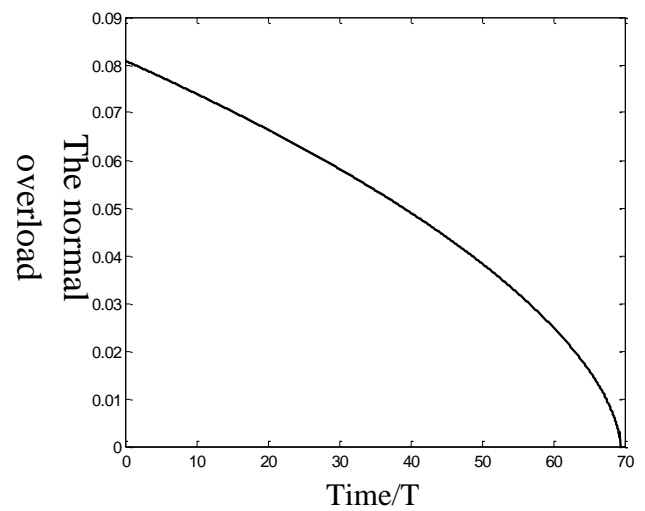

Figure 8. The Required Normal Overload 


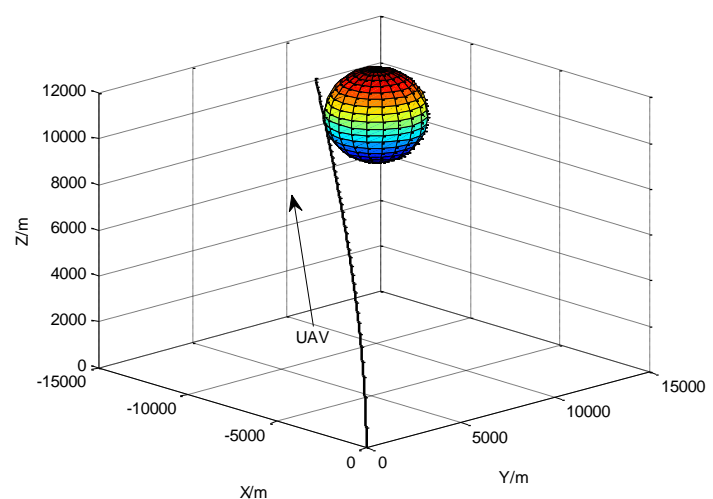

Figure 9 Movement of the UAV in 3-Dspace

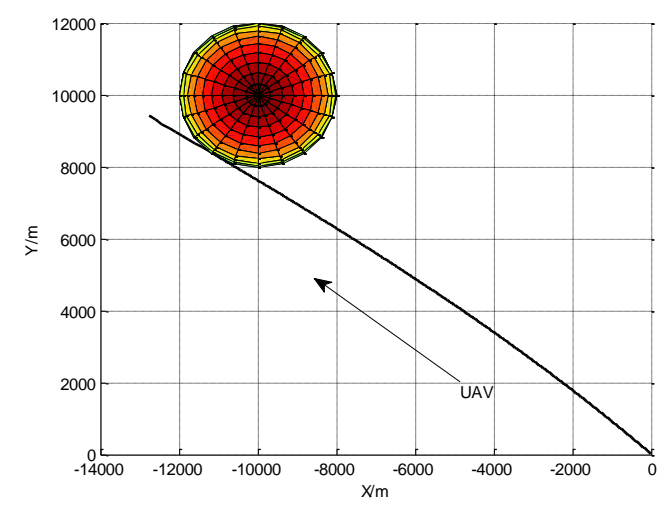

Figure 10 Movement on the Horizontal Plane

From Figure 7 Avoidance vector angle and the heading angle $\psi_{\text {uav }}$, we know the time of heading angle $\psi_{\text {uav }}$ converging to the collision avoidance vector is $t \approx 70 \mathrm{~s}$, Which is roughly equal to the theoretical longest completion-time of collision avoidance $t_{\max } \approx 70 \mathrm{~s}$, The result proves the completion-time of collision avoidance is longest when the proportional navigation coefficient is minimum, which is $N=2.6$.

From Figure 8 The required normal overload, we know the normal overload meets the constraint of the UAV maneuver performance and converges to zero when completing collision avoidance.

From Figure 9 and Figure 10, we know the UAV is just tangent to the obstacle when meets each other, so it ensures that collision avoidance is completed successfully.

(2) The simulation results under the condition of the largest normal overload, which is $N_{\max }=165$, are as follows. 


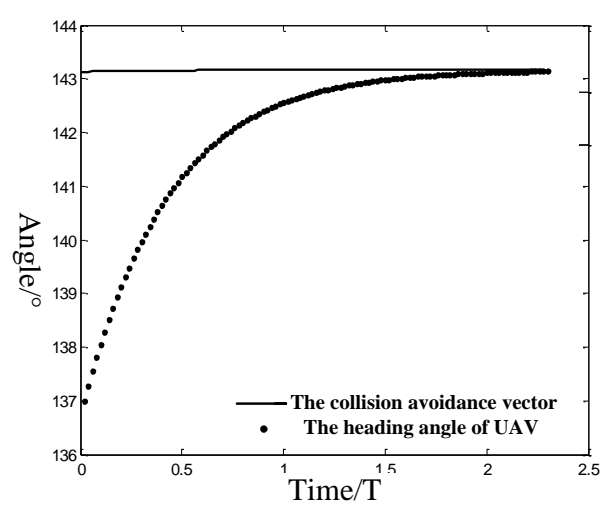

Figure 11. Avoidance Vector Angle and the Heading Angle

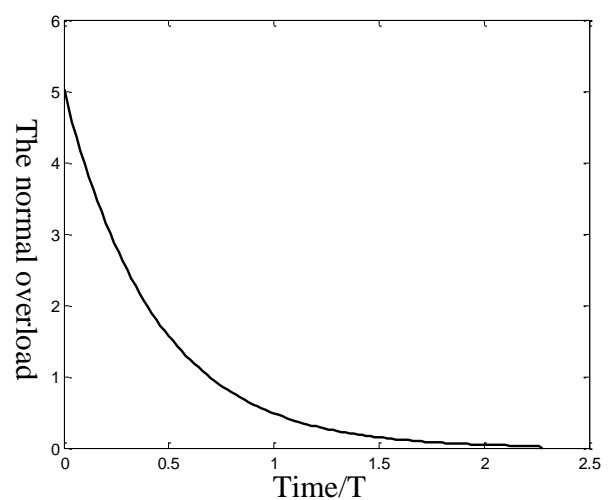

Figure 12. The Required Normal Overload

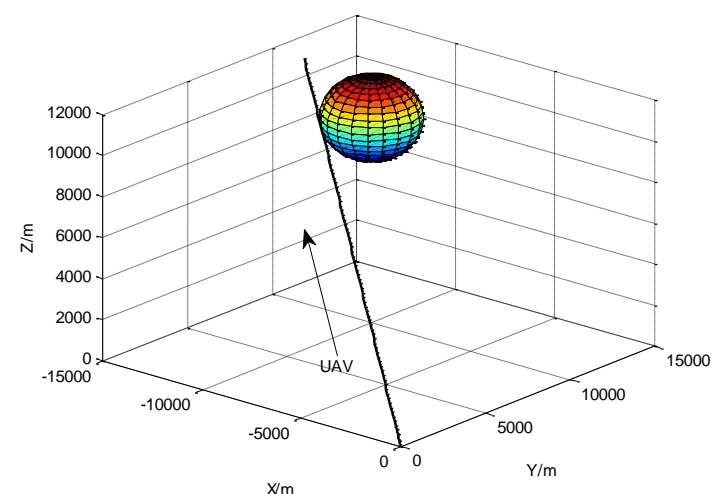

Figure 13. Movement of the UAV in 3-Dspace 


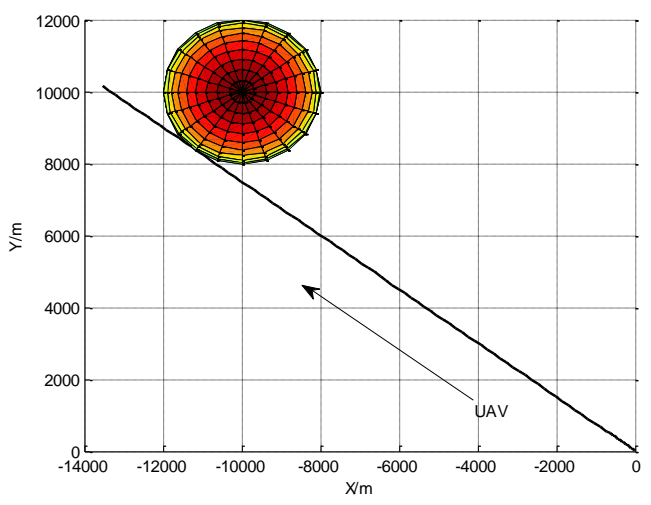

\section{Figure 14. Movement on the Horizontal Plane}

From Figure 11, we know the heading angle $\psi_{\text {uav }}$ converges to the collision avoidance vector when $t=2.3 \mathrm{~s}$, at when the completion-time of collision avoidance is shortest.

From Figure 12, the normal overload is largest at the initial time, which is $n=5$, and monotonically reduced to zero with time, it proves the proportional navigation coefficient $N=165$ is maximum and meets the constraint of the largest normal overload.

From Figure 13 and Figure 14, we know the UAV is just tangent to the obstacle when meets each other, so it ensures that collision avoidance is completed successfully.

\section{Summary}

In this chapter, we suggest to solve the problem of collision avoidance of the UAV to the moving obstacles based on the proportional navigation guidance law. First, we define the collision avoidance vector, then under the acceleration command, we guide the relative velocity vector of the UAV to the collision avoidance vector to complete collision avoidance. Considering the constraints of the maneuver performance of the UAV and the completion-time of collision avoidance, we give the formulations of the required normal overload and the proportional navigation coefficient. Under actual situations, by solving the formulations, we can get the range of proportional navigation coefficient $N$. Moreover, considering the navigation environment of the UAV is three dimensional space, so this method is generalized to the three dimensional space. Simulation results demonstrate the effectiveness of the algorithm. Designing this algorithm, we only need the parameters of the location and velocity of obstacles and UAV, and such parameters can be obtained by airborne radar easily, so the algorithm is operated more convenient.

\section{Acknowledgements}

This research is supported by Aeronautical Science Foundation of China under Grant No20135584010.

\section{References}

[1] O. Khatib, "Real Time Collision Avoidance for Manipulators and Mobile Robots", The International Journal of Robotics Research, vol. 5, no. 1, (1986), pp. 90-98.

[2] G. Parkm and C. Leem, "Artificial potential field based path planning for mobile robots using a virtual obstacle concept", IEEE/ASME International Conference on Advanced Intelligent Mechatronics (AIM), vol. 26, no. 2, (2003), pp. 735-740.

[3] H. S. Shin, B. A. White and A. Tsourdos, "UAV Conflict Detection and Resolution for Static and 
Dynamic Obstacles”, Proceedings of AIAA GNC 2008, Honolulu, HI, AIAA, (2008)-6521.

[4] H. S. Shin, A. Tsourdos and B. White, "UAS Conflict Detection and Resolution Using Differential Geometry Concepts", Sense and Avoid in UAS: Research and Applications, First Edition, Edited by Plamen Angelov. John Wiley \& Sons, Ltd, (2012).

[5] B. A. White, H. S. Shin and A. Tsourdos, "UAV Obstacle Avoidance Using Differential Geometry Concepts", IFAC World Congress 2011, Milan, Italy, (2011), pp. 6325-6330.

[6] F. Belkhouche and B. Bendjilali, "Reactive Path Planning for 3-D Autonomous Vehicles", IEEE Transactions on Control Systems Technology, vol. 20, no. 1, (2012), pp. 249-256.

[7] J. V. D. Berg, M. Lin and D. Manocha, "Reciprocal velocity obstacles for real-time multi-agent navigation”, 2008 IEEE International Conference on Robotics and Automation, (2008), pp. 19281935.

[8] S. C. Han and H. Bang, "Proportional Navigation-Based Optimal Collision Avoidance for UAVs", 2nd International Conference on Autonomous Robots and Agents, (2004).

[9] L. Xiaowei and Y. Xiuxia, "Study on Proportional Navigation-Based Collision Avoidance for UAV", Computer Simulation, vol. 32, no. 1, (2015), pp. 34-39.

\section{Authors}

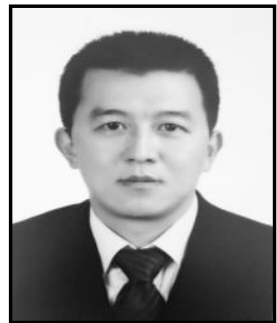

Xiong Zhiyong, he was born in China in 1972. He received his master degree in Northwestern Polytechnical University, Xi'an, China in 1997. Since 1997, he has been Science and Technology on Avionics Integration Laboratory, Shanghai, where he is currently a research professor. His main research interest is integrated avionics system.

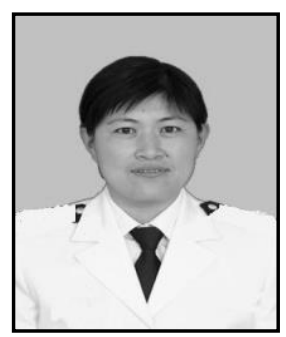

Yang Xiuxia, she was born in Laizhou, Shandong Province, China in 1975. He received his Ph.D. degree in electrical engineering from naval university of engineering, Wuhan, China in 2005. Since 2000, he has been with department of control engineering of naval aeronautical and astronautical university, where he is currently a vice professor. His main research interests include nonlinear control theory with applications to robots, aircraft and other mechanical systems.

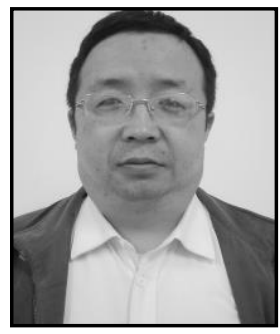

Zhang Yi, he was born in Rongcheng, Shandong Province, China in 1971. He received his master degree in control theory and application from naval aeronautical and astronautical university, Yantai, China in 2001. Since 2000, he has been with department of control engineering of naval aeronautical and astronautical university, where he is currently a vice professor. His main research interests include nonlinear control theory with applications to robots, aircraft and other mechanical systems. 
International Journal of Future Generation Communication and Networking Vol. 9, No.8, (2016) 